\title{
Phosphate starvation enhances phagocytosis of Mycobacterium bovis/BCG by macrophages
}

\author{
Patricia Espinosa-Cueto, Alejandro Magallanes-Puebla and Raul Mancilla* (D)
}

\begin{abstract}
Background: Tuberculosis is an important health problem worldwide. The only available vaccine is $M$. bovis/BCG, an attenuated mycobacterium that activates the innate and the acquired immune system after being phagocytosed by macrophages and dendritic cells. Vaccination fails to prevent adult pulmonary tuberculosis although it may have a protective effect in childhood infection. Understanding how BCG interacts with macrophages and other immunocompetent cells is crucial to develop new vaccines.

Results: In this study we showed that macrophages phagocytose M. bovis/BCG bacilli with higher efficiency when they are cultured without phosphate. We isolated mycobacterial membranes to search for mycobacterial molecules that could be involved in these processes; by immunoblot, it was found that the plasma membranes of phosphatedeprived bacilli express the adhesins PstS-1, LpqH, LprG, and the APA antigen. These proteins are not detected in membranes of bacilli grown with usual amounts of phosphate.

Conclusions: The interest of our observations is to show that under the metabolic stress implied in phosphate deprivation, mycobacteria respond upregulating adhesins that could improve their capacity to infect macrophages. These observations are relevant to understand how M. bovis/BCG induces protective immunity.
\end{abstract}

Keywords: Tuberculosis, M.bovis/BCG, Vaccine, Adhesins, Macrophages

\section{Background}

Tuberculosis (TB) remains an important health problem worldwide. In 2017, there were 10.4 million new cases and 1.7 million deaths [1]. Pulmonary TB is initiated when inhaled bacilli reach the alveoli, the terminal part of the respiratory tract, where they encounter host cells, especially macrophages (MO), the best-equipped cell to contend with intracellular microbes $[2,3]$. After phagocytosis, mycobacteria can be lysed within the phagolysosome, although virulent bacilli have developed the ability to avoid destruction impeding the fusion of the phagosome with the lysosome, thus transforming the $\mathrm{MO}$ in a

\footnotetext{
* Correspondence: mancilla@biomedicas.unam.mx

Departamento de Inmunología, Instituto de Investigaciones Biomédicas, Universidad Nacional Autónoma de México, Circuito Escolar S/N, Ciudad Universitaria, 04510 México City, Mexico
}

friendly niche where they proliferate and persist $[2,4]$. In an initial step, Mycobacterum tuberculosis (Mtb) binds alveolar MOs through mycobacterial adhesins or opsonins that interact with a variety of receptors, including CR1, CR3, and CR4, IgGFc, surfactant A protein and C-type lectins [3]. Information on the repertoire of Mtb adhesins is scanty; a few have been characterized including lipoarabinomannan, a cell wall glycolipid [4], LpqH, a $19-\mathrm{kDa}$ surface located lipoprotein [5], the heparinbinding hemagglutinin [6], PE_PGRS33, a member of the PE-PGRS family [7], LprG a 25-kDa glycolipoprotein [8], APA a 45-47-kDa mannose-containing glycoprotein [9] and PstS-1, a phosphate transporter [10]. It is important to know better the repertoire of mycobacterial adhesins since it could be helpful to design novel strategies for TB prevention and treatment [11].

(c) The Author(s). 2020 Open Access This article is licensed under a Creative Commons Attribution 4.0 International License, which permits use, sharing, adaptation, distribution and reproduction in any medium or format, as long as you give appropriate credit to the original author(s) and the source, provide a link to the Creative Commons licence, and indicate if changes were made. The images or other third party material in this article are included in the article's Creative Commons licence, unless indicated otherwise in a credit line to the material. If material is not included in the article's Creative Commons licence and your intended use is not permitted by statutory regulation or exceeds the permitted use, you will need to obtain permission directly from the copyright holder. To view a copy of this licence, visit http://creativecommons.org/licenses/by/4.0/ The Creative Commons Public Domain Dedication waiver (http://creativecommons.org/publicdomain/zero/1.0/) applies to the data made available in this article, unless otherwise stated in a credit line to the data. 
Recently, it has been reported that PstS-1, the $38-\mathrm{kDa}$ glycoprotein, binds the MO mannose receptor triggering the phagocytosis of mycobacteria [10]. PstS-1 has focused much interest as a phosphate $(\mathrm{Pi})$ transporter whose expression is upregulated under $\mathrm{Pi}$ starvation conditions [12-14]. Pi deprivation can activate the expression of a variety of genes not involved in phosphate metabolism that participate in the pathogenesis of TB [15]. Herein, we show that $M$. bovis/BCG bacilli grown without phosphate are engulfed with increased efficiency by $\mathrm{MO}$, an event that was associated with decreased phagosome acidification. Considering that $\mathrm{Pi}$ starvation increases the surface expression of PstS-1, we searched its expression in isolated plasma membranes; we found that together with PstS-1, the membranes of Pi-deprived mycobacteria express the mycobacterial adhesins $\mathrm{LpqH}$, LprG, and the APA antigen, glycoproteins that are not directly involved in phosphate regulation.

\section{Results}

Mycobacteria cultured under $\mathrm{Pi}$ deprivation conditions are phagocytosed with higher efficiency by macrophages The phagocytosis assays were carried out incubating J774A.1 macrophage-like cells with mycobacteria labeled with the lipophilic red fluorescent dye PKH-26 at 1:2, 1: 5 and 1:20 MOI. Immunofluorescence microscopy revealed that the number of MOs with engulfed or bound bacilli was significantly higher with Pi-deprived mycobacteria (Fig. 1a, b). Phagocytosis was quantitated by fluorescence activated cell sorting (FACS) with PKH-26 a

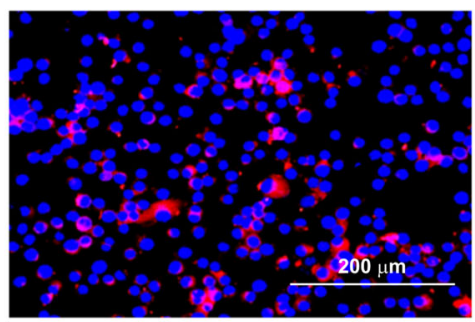

$\mathrm{Pi+}$

C
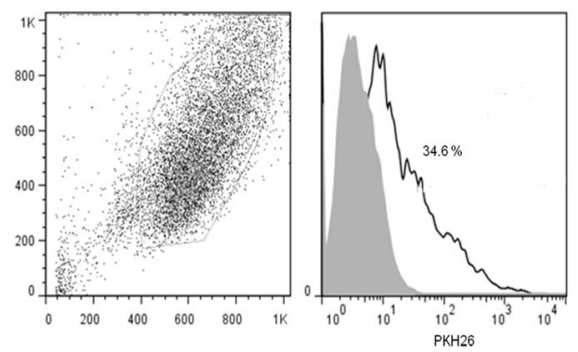

e

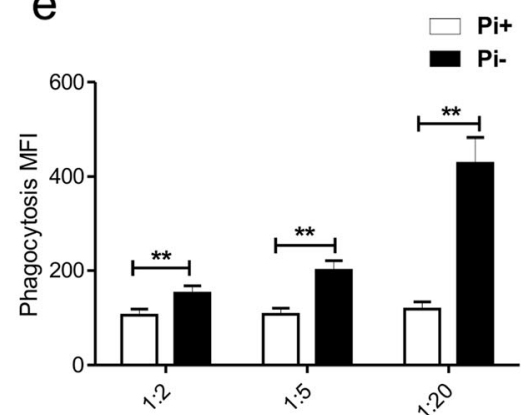

b

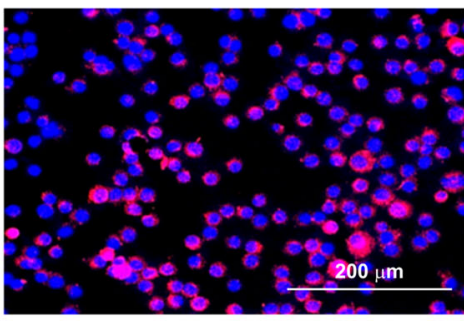

Pi-

d

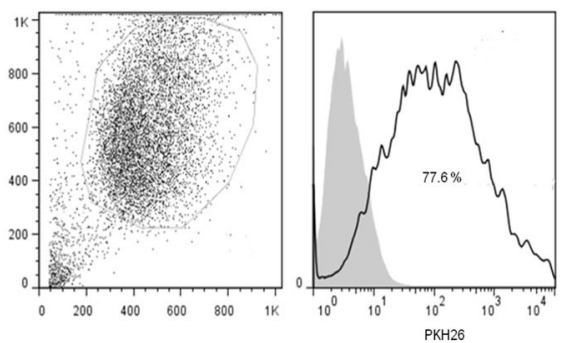

f

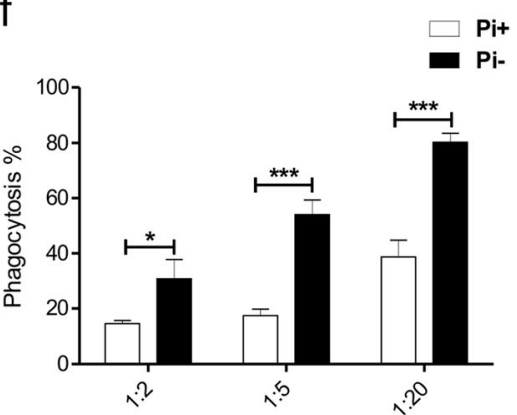

Fig. 1 Phosphate deprivation increases M. bovis/BCG phagocytosis. J774A.1 MOs $\left(5 \times 10^{5}\right)$ were incubated with M. bovis/BCG bacilli labeled red with the PKH-26; nuclei were stained blue with DAPI. Immunofluorescence microscopy shows a marked difference in the degree of phagocytosis between bacilli grown with and without $\operatorname{Pi}(\mathbf{a}, \mathbf{b})$. For FACS analysis gates considering the percent and the mean fluorescence index (MFI) were carried out (c, d). Dose-dependent phagocytosis that was significantly higher with Pi-deprived mycobacteria was observed (e, $\mathbf{f}$ ). At a 1:20 MOI, 80.2\% of MOs phagocytosed bacilli cultured without Pi while the percent phagocytosis of bacilli cultured with Pi was 38.7; $p<0.05$; unpaired $t$ Student's test. Results of 5 independent experiments are shown 
stained bacilli (Fig. 1c, d). Phagocytosis expressed in mean fluorescence intensity (MFI) and percent was dose-dependent and considerably higher with $\mathrm{Pi}$ deprived bacilli (Fig. 1e, f). At a 1:20 MOI, the percent of phagocytosis of bacilli grown without $\mathrm{Pi}$ was 80.2 and $38.7 \%$ of bacilli grown with usual amounts of Pi.

\section{Assays to verify the viability of phagocytosed mycobacteria grown with and without phosphate} After $4 \mathrm{~h}$ of MO phagocytosis at 1:10 and 1:20 MOI, the viability of ingested bacilli was assessed using the LIVE/ DEAD Baclight bacterial viability Kit (Molecular Probes, Invitrogen, Carlsbad, CA EUA) following manufacturer's instructions. Bacilli were fixed in $4 \%$ paraformaldehyde for $20 \mathrm{~min}$ and rinsed. According to manufacturer's protocol, viability analysis was carried out by FACS separating bacilli in two regions of the log-integrated red fluorescence propidium iodide versus Syto 9; the number of bacilli found within these regions were used to estimate the percentage of viability (Fig. 2a). The results showed that at 1:10 MOI the viability of the Pi-deprived bacilli was significantly increased (Fig. 2b).

\section{High phagocytosis of microbeads coated with plasma} membrane proteins of phosphate-deprived mycobacteria To study the role of mycobacteria proteins in the phagocytosis of bacilli grown under Pi starvation conditions, we carried out assays with fluorescent microbeads coated with proteins obtained from plasma membranes isolated from whole mycobacteria grown with and without Pi. For phagocytosis assays, MOs $\left(5 \times 10^{6}\right)$ cultured in RPMI 1640 with $10 \%$ heat-inactivated fetal bovine serum were placed on acid-washed glass coverslips. Microbeads were added to cells at 1:5, 1:20 and 1:100 rate for $4 \mathrm{~h}$ at $37^{\circ} \mathrm{C}$ in $5 \% \mathrm{CO}_{2}$. To quantitate phagocytosis of microbeads, the slides were analyzed with a Nikon epifluorescence microscope examining at least 400 cells in random fields of 3 slides at each experimental time (Fig. 3a, b). MOs were scored positive regardless of the number of bound or engulfed microbeads. Immunofluorescence microscopy of 400 or more cells showed that binding/ phagocytosis of microbeads was dose-dependent and significantly higher with microbeads coated with membrane proteins obtained from bacilli grown without Pi (Fig. 3c); this difference was evident in assays incubating cells at 1 : 20 and 1:100 macrophage/microbead rates.

\section{Phagosome acidification in macrophages that have engulfed phosphate-deprived mycobacteria}

An outstanding ability of virulent mycobacteria is to inhibit phagosome acidification and phagolysosome fusion [2]. We studied the phagosomal acidification process by immunofluorescence microscopy using $M$. bovis/BCG grown with or without Pi labelled green with fluorescein isothiocyanate (FITC) and MOs labelled with LysoTracker, a marker that turns red at low pH (Fig. 4a). Bacilli located in Lysotracker positive vacuoles were yellow fluorescent due to overlapping of FITC and the red fluorescence of Lysotracker. The engulfed bacilli emitted a FITC green fluorescence when were located in Lysotracker negative vacuoles (Fig. 4a). When infected with bacilli grown with usual amounts of $\mathrm{Pi}, 80.75 \%$ of cells were Lysotracker positive and with Pi-deprived bacilli the percent of Lysotracker labeled MOs was reduced to $73.06 \%$ FACS analysis of the phagocytosis assays confirmed the immunofluorescence findings showing that Pi-deprived bacilli phagocytosis is associated with decreased Lysotracker labeling (Fig. 4b, c).
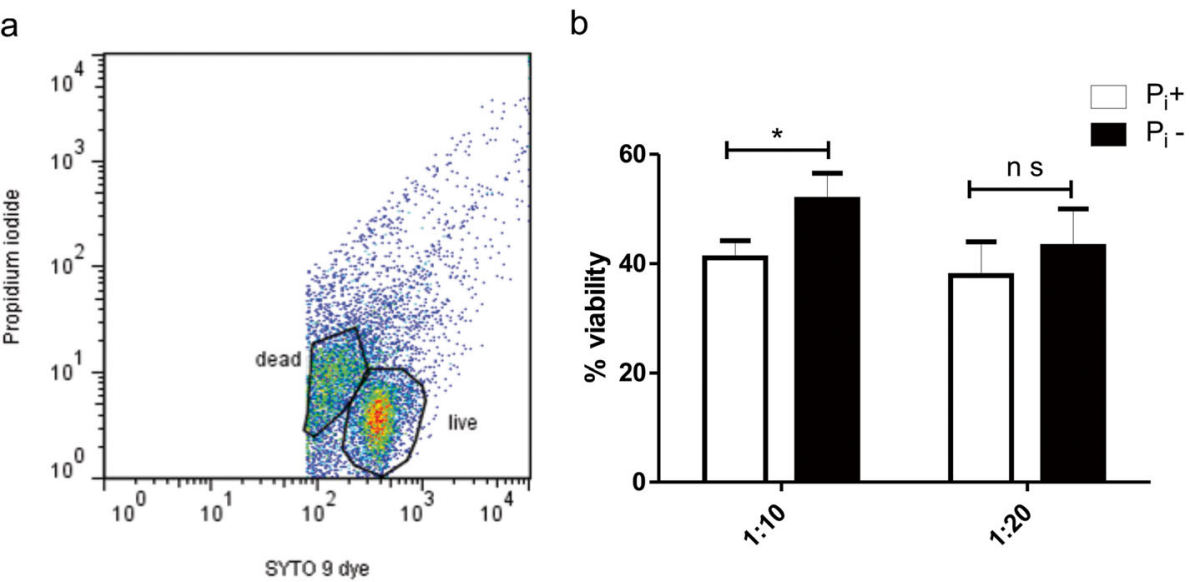

Fig. 2 Phosphate deprivation increases the viability of mycobacteria within macrophages. After $4 \mathrm{~h}$ phagocytosis, mycobacteria viability was analyzed by FACS using the LIVE/DEAD viability assay. A dot plot was set to differentiate living from dead mycobacteria (a). The survival rate was calculated using the formula: \% viability = \# of bacteria in live region / \# of bacteria in dead region $\times(100)(\mathbf{b}) . p<0.05$ Mann Whitney test. Results of 3 experiments are presented 
a

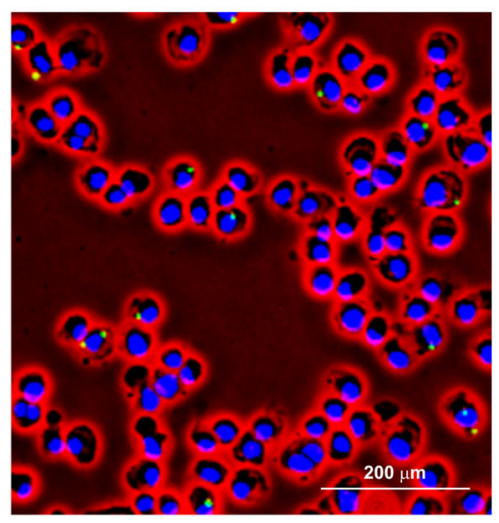

$\mathrm{P}_{\mathrm{i}}^{+}$ b

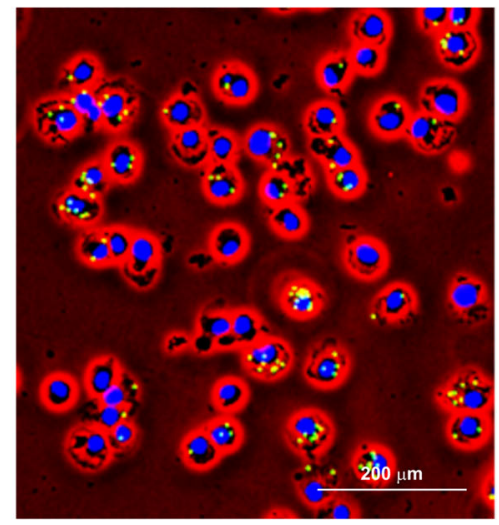

$\mathrm{P}_{\mathrm{i}-}^{-}$

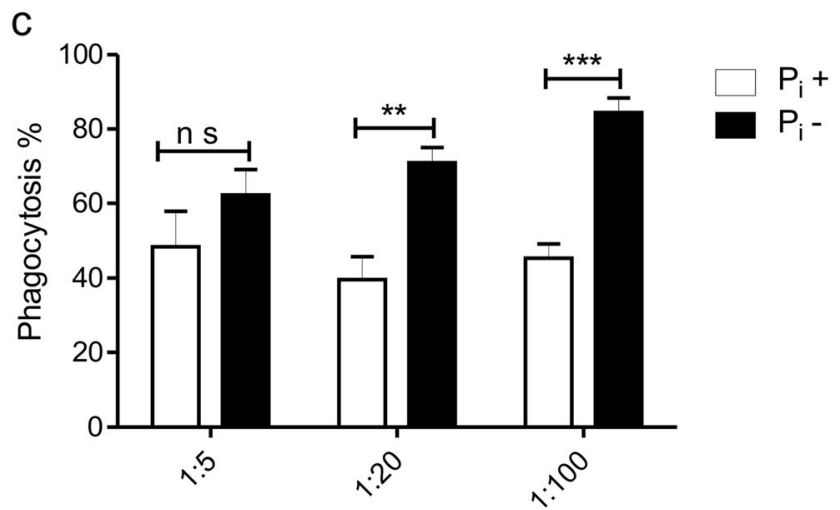

Fig. 3 Phagocytosis of membrane protein-coated microbeads is higher with phosphate deprived bacilli proteins. Assays were carried out with J774A.1 macrophage-like cells and green fluorescent microbeads coated with plasma membrane proteins obtained from mycobacteria grown with and without Pi. MOs $\left(5 \times 10^{5}\right)$ were placed on acid-washed glass coverslips. Microbeads were added to cells at a 1:5, 1:20 and 1:100 rate for $4 \mathrm{~h}$ at $37^{\circ} \mathrm{C}$ in $5 \% \mathrm{CO}_{2}$. The percentage of cells with ingested or bound microbeads was estimated counting at least $400 \mathrm{MOs}(\mathbf{a}, \mathbf{b})$. At 1:100 rate the phagocytosis of microbeads coated with proteins of Pi-deprived bacilli was $84.44 \%$ and of bacilli grown with Pi it was $45.4 \%$ (a, c). $p<0.05$; unpaired $t$ Student's test. Results of 5 experiments are presented

\section{Phosphate-starved mycobacteria increment the} expression of adhesins in isolated plasma membranes We have previously shown by immunoelectron microscopy that PstS-1 is a cell surface located adhesin that triggers the phagocytosis of mycobacteria [10]. To study its location in Pi- starved mycobacteria, we isolated plasma membranes to carry out 1-D electrophoresis and immunoblot (Fig. 5). By Coomasie blue staining and immunoblot with a polyclonal anti- $M$. bovis/BCG antiserum the number of protein bands was higher in membranes of Pi-deprived mycobacteria. In the isolated plasma membranes immunobloting with $\mathrm{mAb}$ available in our laboratory that recognizes adhesins that participate in the phagocytosis of mycobacteria 5 reactive bands of $38,19,23$, and $47 \mathrm{kDa}$ that correspond to PstS1, LpqH, LprG, and the APA antigen, respectively (Fig. 5). In the membranes of bacilli grown with Pi band mild APA reactivity was observed.

\section{Discussion}

In this study, we observed that $M$. bovis/BCG cultured without $\mathrm{Pi}$ acquire properties that could indicate increased virulence. First, bacilli were phagocytosed by MOs with high efficiency, a property suggesting increased infectivity. These changes were associated with an increase in cell protein band expression in cell membranes. Morever, in isolated plasma membranes of $\mathrm{Pi}$ deprived bacilli, it was demonstrated the expression of glycoproteins that have been characterized as adhesins that play a critical role in the infection of host cells.

It is noteworthy that mycobacteria subjected to the metabolic stress implied in Pi deprivation increment the expression of adhesins which are virulence molecules that bind to complementary molecules present in the surface of MOs and other host cells [11]. Information on the repertoire of Mtb adhesins is needed since it could help to design prevention and treatment strategies aimed 
a

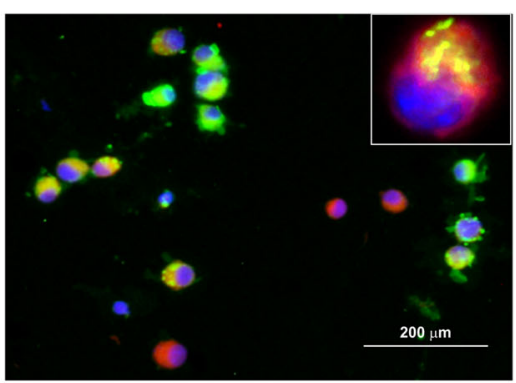

b

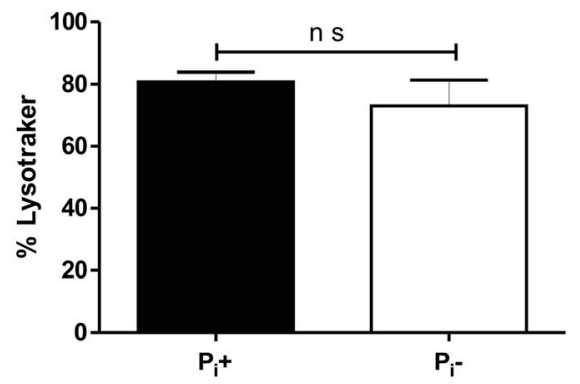

C

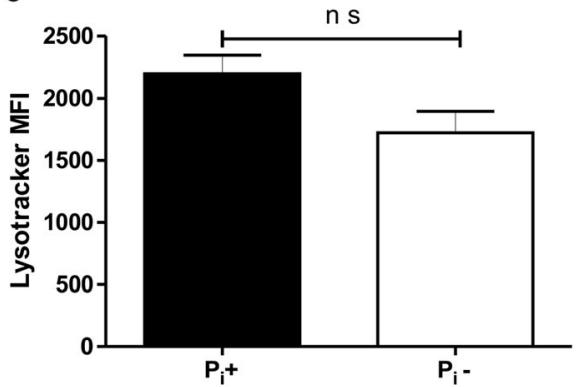

Fig. 4 Phagosome acidification in MOs that engulfed mycobacteria grown without phosphate. Phagocytosis assays were performed with MOs labelled with LysoTracker, whose red fluorescence intensifies at low pH, and FITC-labelled bacilli cultured with or without Pi. By immunofluorescence microscopy at least $400 \mathrm{MO}$ were counted. $\mathrm{MO}$ emitting red fluorescence were scored as Lysotracker positive. The immunofluorescence patterns are shown; bacilli located in non-acidified compartments (LysoTracker negative) are green fluorescent. In acidified vacuoles (LysoTracker positive) bacilli emitted yellow fluorescence due to overlapping of FITC-labelled bacilli and Lysotracker red fluorescence (insert), (a). It was found that $80.75 \%$ of MOs incubated with mycobacteria grown with usual amount of Pi were LysoTracker positive while incubation with Pi-deprived bacilli $76.06 \%$ of MO were Lysotracker labelled (b), a difference that was not statistically significant ( $\mathrm{n}$ s; MannWhitney Test). Four independent experiments were performed. FACS showed that the mean fluorescence index (MFI) was less in MO that phagocytosed Pi-deprived mycobacteria (c). This difference was not statistically significant (n s; Mann-Whitney Test)
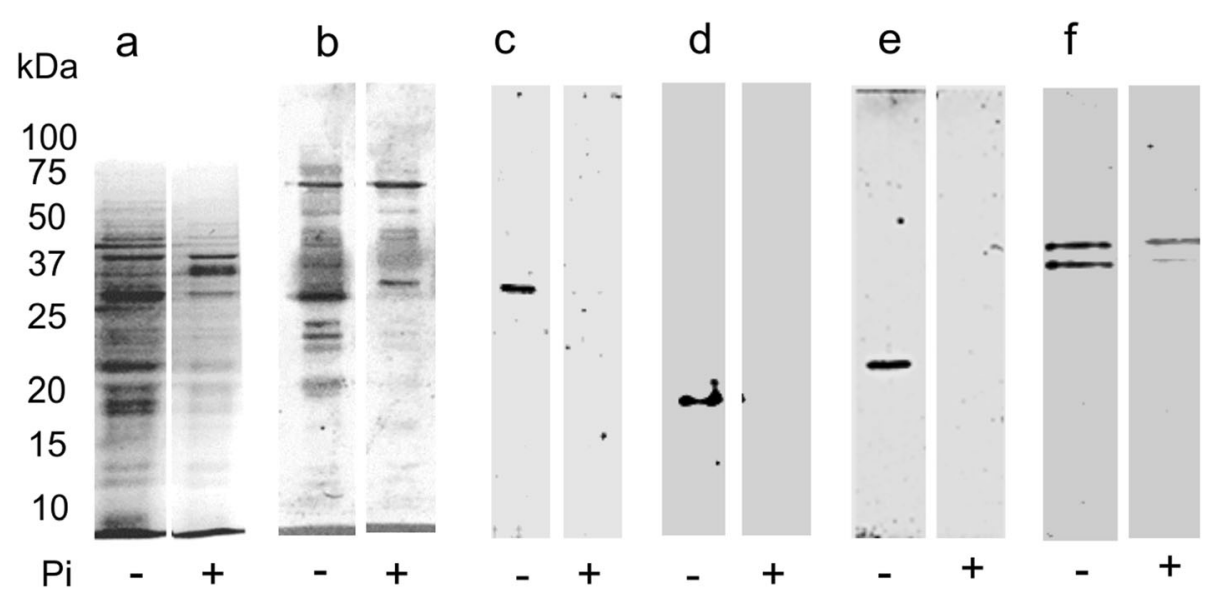

Fig. 5 Cell membrane located PstS-1, LpqH, LprG, and APA are expressed by Pi-deprived mycobacteria. M.bovis/BCG bacilli were cultured with (+) and without $\mathrm{Pi}(-)$ for $24 \mathrm{~h}$ and the membranes were isolated by sonication and centrifugation, separated by $12 \%$ SDS-PAGE and stained with Coomasie blue (a). The membranes were transferred to PVDF for immunoblot with an anti-M.bovis/BCG antiserum which detected many protein bands more numerous in membranes of Pi-deprived bacilli (b). Using Abs available in our laboratory the expression of PstS-1, LpqH, LprG, and APA was demonstrated in isolated plasma membranes of Pi-deprived mycobacteria (c-f) 
to block the crucial step that is the infection of host cells by the mycobacteria. A few mycobacterial adhesins have been characterized including PstS-1, the $38-\mathrm{kDa}$ antigen which is high-affinity protein that transports $\mathrm{Pi}[10,13]$. It has been recognized its high antigenicity at the humoral level that allowed the design of diagnostic serologic tests [16-19]. PstS-1 was characterized as a glycoprotein with mannose residues and more recently its role of adhesin in the phagocytosis of mycobacteria was recognized [10]. PstS-1 has focused much interest as a high-affinity Pi transporter that carries Pi from the periplasm to the cytoplasm. It has been reported that $\mathrm{Pi}$ starved mycobacteria upregulates PstS-1 which is $30 \%$ similar in amino acid sequence to PstS-1 from Escherichia coli [12].

Our current findings suggesting increased virulence in mycobacteria subjected to Pi deprivation are in keeping with previous studies [20]. Pst genes are overexpressed in mycobacteria grown under Pi starvation conditions; mutations in these genes affect the import of $\mathrm{Pi}$ and reduces the proliferation of mycobacteria inside macrophages and in experimentally infected mice [21, 22]. On the other hand, it has been observed that Mtb Pst system neutralizes IFN-gamma-dependent host immunity [22].

There is much information about the role membrane proteins of Mtb and other microbes play in the pathogenesis of infection [23]; their strategic location at the microbe surface allow their efficient interaction with host cells to promote phagocytosis and when they are antigenically active an immune response that can be cell or antibody-mediated [23]. Mycobacterial membrane proteins may also exert inhibitory effects on host cell activity. In this study we found that in addition to PstS-1, Pi-deprived mycobcteria express membrane proteins that are considered adhesins involved in the pathogenesis of the mycobacterial infection. $\mathrm{LpqH}$, the $19 \mathrm{kDa}$ glycolipoprotein, participates in MO infection as an adhesin that binds the mannose receptor triggering the phagocytosis of bacilli and downregulating MHC-II antigen presentation interfering with TLR2 and MAPK signaling [22]. The APA antigen plays a prominent role in Mtb virulence; it functions as a mycoyl-transferase that catalyzes the attachment of mycolic acids to arabinogalactan and the biogenesis of cord factor, a very active virulence factor [24]. APA is expressed at the bacterial surface, which explains its interaction with fibronectin, elastin and the surfactant protein A. LprG, the $25-\mathrm{kDa}$ glycolipoprotein transports ManLAM to the mycobacterial cell-wall and as an adhesin it may bind the MO mannose receptor facilitating phagocytosis and inhibition of the phagosome-lysosome fusion. Isolated LprG was found to inhibit MHC-II Ag processing by THP-1 cells and primary human $\mathrm{MO}$ through prolonged TLR2 activation $[25,26]$.

\section{Conclusions}

The response of $\mathrm{M}$. bovis/BCG to Pi deprivation could be of interest for vaccine design. The increased phagocytosis and intracellular viability of phosphate deprived bacilli could favor the activation of immunocompetent cells. The fact that phagosome acidification is virtually preserved would favor the lysis of mycobacteria and the generation of antigenic peptides which are needed to induce an effective immune response. To gain insight in the meaning of our findings, studies with dendritic cells, the master antigen presenting cell, are required.

\section{Methods}

\section{Antibodies}

Monoclonal antibodies to LpqH and PstS-1 were obtained from BEI Resources (Manassas, VA, USA). A monoclonal antibody to Apa was developed in our laboratory. A polyclonal antiserum to LprG was donated by Dr. Clara Espitia (Universidad Nacional Autónoma de México, Mexico City).

\section{Culture of Mycobacterium bovis/BCG under phosphate deprivation conditions}

The method of Braibant et al. was followed [14]. Briefly, $M$. bovis/BCG was cultured in Sauton medium with $\mathrm{K}_{2} \mathrm{HPO}_{4}(0.5 \mathrm{~g} / \mathrm{L})$ at $37^{\circ} \mathrm{C}$, until optic density of 460 was reached. Thereafter, aliquots were taken and inoculated in Sauton medium that was supplemented with an excess of $\mathrm{K}_{2} \mathrm{HPO}_{4}$ to a final concentration of $3 \mathrm{~g} / \mathrm{L}$; the mycobacteria were cultured at $37^{\circ} \mathrm{C}$ until optic density of 460 was reached. After that, two aliquots were rinsed, centrifuged at $6000 \mathrm{~g}$, and inoculated in Sauton medium. One aliquot was cultured without $\mathrm{K}_{2} \mathrm{HPO}_{4}$ and the other with $0.5 \mathrm{~g} / \mathrm{L} \mathrm{K}_{2} \mathrm{HPO}_{4}$. After $24 \mathrm{~h}$ of culture, bacilli were collected, rinsed with TBS and frozen until use.

\section{Phagocytosis assays with phosphate-deprived mycobacteria}

We carried assays with mycobacteria that were grown with and without Pi as described above. The Balb/c-derived murine macrophage-like tumor cell line J774A.1 was obtained from the American Type Culture Collection (Manassas, VA, USA). Cells were cultured in RPMI 1640 supplemented with $10 \%$ fetal calf serum. MOs $(5 \times$ $10^{5}$ ) were incubated with PKH26 labeled bacilli at 1:2, 1 : 5, and 1:20 MOI, at $37^{\circ} \mathrm{C}$ for $4 \mathrm{~h}$. After extensive rinsing with PBS, the cells were fixed in $4 \%$ paraformaldehyde in PBS for $20 \mathrm{~min}$. For immunofluorescence microscopy, cytospin slides were mounted with ProLong Antifade with DAPI (Invitrogen, Eugene, OR, USA) and examined with a Nikon epifluorescence microscope. Phagocytosis was analyzed by FACS. At least 10,000 cells were analyzed in a FACSClibur (Becton Dickinson, San Jose CA, USA) operating with FlowJo 7 software and a $488 \mathrm{~nm}$ 
argon laser. The gates were set following established forward and side scatter parameters.

Analysis of the mycobacterial viability after phagocytosis Assays to verify the viability of phagocytosed mycobacteria were carried out. After $4 \mathrm{~h}$ phagocytosis of mycobacteria at 1:10 and 1:20 MOIs, MOs were recovered in TBS, lysed with $0.1 \%$ sodium deoxycholate for $5 \mathrm{~min}$, and centrifuged twice at 12,000 rpm for $10 \mathrm{~min}$. Thereafter, to assess viability the LIVE/DEAD Baclight bacterial viability Kit (Molecular Probes) was used following the manufacturer instructions. Afterward, bacilli were fixed in $4 \%$ paraformaldehyde for $20 \mathrm{~min}$, rinsed in TBS and analyzed by flow cytometry. Because both live and dead cells exhibit green fluorescence, the log-integrated green fluorescence was adjusted to eliminate debris. Populations of bacteria were discriminated as two regions of the log-integratedred fluorescence, and the numbers of bacteria found within these regions were used to estimate the percentage of viable organisms in the population.

\section{Binding/phagocytosis assays with microbeads coated with mycobacterial membrane proteins}

We assessed a possible role of mycobacterial membrane proteins in the phagocytosis of mycobacteria employing green fluorescent Polystyrene $1 \mu \mathrm{m}$ microbeads (Molecular Probes, Eugene, OR, USA) coated with plasma membrane proteins obtained from mycobacteria grown with and without $\mathrm{Pi}$. Microbeads and $500 \mu \mathrm{g}$ membrane proteins were mixed in $1 \mathrm{~mL}$ MES Buffer 2-[N-morpholino] ethane sulfonic acid (pH 6). After that, 1-ethyl-3-(3dimethylamine nopropyl)-carbodiimide was added to a $2.5 / \mathrm{ml}$ final concentration. After overnight incubation, $100 \mathrm{mM}$ glycine was added to stop the reaction. To block remaining reactive sites, microbeads were rinsed with PBS and kept at $4{ }^{\circ} \mathrm{C}$ in PBS containing $1 \%$ BSA. For phagocytosis assays, $5 \times 10^{5}$ MOs were cultured in RPMI 1640 with 10\% heat-inactivated FBS and placed on acid-washed glass coverslips. Microbeads were added to cells at a $1: 20$ rate and incubated for $4 \mathrm{~h}$ at $37^{\circ} \mathrm{C}$ in $5 \% \mathrm{CO}_{2}$. The slides were rinsed in PBS to remove nonadherent microbeads and fixed in 1\% paraformaldehyde for $10 \mathrm{~min}$.

\section{Phagosome maturation after phagocytosis of mycobacteria}

For these analyses bacilli grown with and without PI were labeled green with fluorescein isothiocyanate (FITC). Briefly, $50 \mu \mathrm{l}$ of a bacilli suspension was suspended in $950 \mu \mathrm{l}$ carbonate buffer $0.1 \mathrm{M}, \mathrm{pH} 9$ and 0.5 mg FITC were added for $2 \mathrm{~h}$. To finish labeling the mycobacteria were centrifuged at $10,500 \mathrm{~g}$ for $10 \mathrm{~min}$ and rinsed 3 times in PBS. To examine whether $\mathrm{Pi}$ deprived mycobacteria acquired the ability to alter phagosome maturation, we use the acidotropic dye LysoTracker Red DND-99 (Molecular Probes, Eugene, OR). After rinsing, FITC labeled mycobacteria were added for $4 \mathrm{~h}$ at a 1 : 10 MOI. In a final step, LysoTracker was added in RPMI (1:20,000) for $20 \mathrm{~min}$ before phagocytosis was finished. MOs were rinsed, fixed with $4 \%$ paraformaldehyde and placed in glass slides. To verify LysoTracker labeling slides were examined by immunofluorescence microscopy. Besides, we quantitated by FACS the extent of LysoTracker phagosome labeling and the phagocytosis of FITC labeled bacilli.

Isolation of mycobacterial plasma membranes to identify proteins expressed during phosphate deprivation

Mycobacteria were grown with and without Pi as described above. To obtain mycobacterial plasma membranes we followed a published method [27]. Briefly, in a lysis buffer containing protease inhibitors, bacilli were sonicated at $60 \mathrm{kHz}$ in iced water (20 cycles 1 min each) and centrifuged at $1000 \mathrm{~g}$ and the supernatant was centrifuged at 27, $000 \mathrm{~g}$; the supernatant obtained was centrifuged at $100,000 \mathrm{~g}$ to obtain in the pellet the plasma membranes. Protein concentration was measured by the Lowry protein assay (Bio-Rad Laboratories, Hercules, CA, USA). To identify membrane proteins were separated by $12 \%$ SDS-PAGE, stained with Coomassie blue, transferred to PVDF membranes and incubated with a rabbit antiserum to $M$. bovis/BCG. Isolated plasma membranes were identified with a mAb to $\mathrm{LpqH}$ and PstS-1 (donated by BEI Resources (Manassas, VA, USA), and to APA (developed in our laboratory) and with a polyclonal antiserum to LprG (donated by PhD Clara Espitia); bound antibodies were detected with goat antimouse and a goat anti-rabbit IgG antibodies labeled with horseradish peroxidase. The reactive bands were visualized by chemiluminescence with SuperSignal West Dura kit (Pierce, Rockford, IL, USA).

\section{Abbreviations}

TB: Tuberculosis; Mtb: Mycobacterium tuberculosis; BCG: Mycobacterium bovis Calmette Guerin; Pi: Phosphate; FACS: Fluorescence activated cell sorter; MFI: Mean fluorescence index; FITC: Fluorescein isothiocyanate

\section{Acknowledgements \\ Not applicable.}

Authors' contributions

All authors have read and approved the manuscript. PEC acquired, analyzed data and revised the manuscript; AMP acquired, analyzed data and revised the manuscript; RM designed the work, data interpretation and wrote the original manuscript.

\section{Funding}

This work was funded by Programa de Apoyos a Proyectos de Investigación e Inovación Tecnológica No. IN210816 (URL: http://dgapa.unam.mx/index. php/impulso-a-la-investigacion/papiit). The funders had no role in study design, data collection and analysis, decision to publish, or preparation of the manuscript. 


\section{Availability of data and materials}

All data are included in the manuscript.

\section{Ethics approval and consent to participate}

Not applicable.

\section{Consent for publication}

Not applicable.

\section{Competing interests}

The authors declare that they have no competing interests.

Received: 21 November 2019 Accepted: 4 June 2020

Published online: 09 June 2020

\section{References}

1. WHO. Global tuberculosis report 2017. Geneva: WHO Press; 2017.

2. Clemens DL, Horwitz MA. Characterization of the Mycobacterium tuberculosis phagosome and evidence that phagosomal maturation is inhibited. J Exp Med. 1995:181:257-70.

3. Ernst JD. Macrophage receptors for Mycobacterium tuberculosis. Infect Immun. 1998:66:1277-81.

4. Schlesinger LS, Hull SR, Kaufman TM. Binding of the terminal mannosyl units of lipoarabinomannan from a virulent strain of Mycobacterium tuberculosis to human macrophages. J Immunol. 1994;152:4070-9.

5. Diaz-Silvestre H, Espinosa-Cueto P, Sanchez-Gonzalez A, Esparza-Ceron MA Pereira-Suarez AL, Bernal-Fernandez G, et al. The 19-kDa antigen of Mycobacterium tuberculosis is a major adhesin that binds the mannose receptor of THP-1 monocytic cells and promotes phagocytosis of mycobacteria. Microb Pathog. 2005;39:97-107.

6. Menozzi FD, Bischoff R, Fort E, Brennan MJ, Locht C. Molecular characterization of heparin-binding hemagglutinin, a mycobacterial adhesin Proc Natl Acad Sci U S A. 1998:95:12625-1263.

7. Palucci I, Camassa S, Cascioferro A, Sali M, Anoosheh S, Zumbo A, et al. PE_ PGRS33 contributes to Mycobacterium tuberculosis entry in macrophages through interaction with TLR2. PLoS One. 2016. https://doi.org/10.1371/ journal.Pine. 0150800

8. Drage MG, Tsai HC, Pecora ND, Cheng TY, Arida AR, Shukla S, et al Mycobacterium tuberculosis lipoprotein LprG (Rv1411c) binds triacylated glycolipid agonists of toll-like receptor 2. Nat Struct Mol Biol. 2010;17:108895.

9. Ragas A, Roussel L, Puzo G, Riviere M. The Mycobacterium tuberculosis cell surface glycoprotein apa as a potential adhesin to colonize target cells via the innate immune system pulmonary C-type lectin surfactant protein a. J Biol Chem. 2007;282:5133-42.

10. Esparza M, Palomares B, García T, Espinosa P, Zenteno E, Mancilla R. PstS-1, the 38-kDa Mycobacterium tuberculosis glycoprotein, is an adhesin, which binds the macrophage mannose receptor and promotes phagocytosis. Scand J Immunol. 2015;81:46-55.

11. Govender VS, Ramsugit S, Pillay M. Mycobacterium tuberculosis adhesins: potential biomarkers as anti-tuberculosis therapeutic and diagnostic targets. Microbiology. 2014;160:1821-31.

12. Andersen $A B$, Ljungqvist $L$, Olsen $M$. Evidence that protein antigen $b$ of Mycobacterium tuberculosis is involved in phosphate metabolism. J Gen Microbiol. 1990;136:477-80.

13. Espitia C, Elinos M, Hernández-Pando R, Mancilla R. Phosphate starvation enhances expression of the immunodominant 38-kilodalton protein antigen of Mycobacterium tuberculosis: demonstration by immunogold electron microscopy. Infect Immun. 1992:60:2998-3001.

14. Braibant $M$, Lefèvre $P$, De Wit L, Peirs P, Ooms J, Huygen $K$, et al. Content J. A Mycobacterium tuberculosis gene cluster encoding proteins of a phosphate transporter homologous to the Escherichia coli Pst system. Gene. 1996;176:171-6.

15. Santo-Beneit $F$. The Pho regulon: a huge regulatory network in bacteria. Front Microbiol. 2015;6:402. https://doi.org/10.3389/fmicb.2015.00402.

16. Harboe M, Wiker HG. The 38-kDa protein of Mycobacterium tuberculosis: a review. J Infect Dis. 1992;166:874-84.

17. Wilkinson RJ, Hasløv K, Rappuoli R, Giovannoni F, Narayanan PR, Desai CR, et al. Evaluation of the recombinant 38-kilodalton antigen of Mycobacterium tuberculosis as a potential immunodiagnostic reagent. J Clin Microbiol. 1997; 35:553-7.
18. Espitia C, Cervera I, Gonzalez R, Mancilla R. A 38-kD Mycobacterium tuberculosis antigen associated with infection. Its isolation and serologic evaluation. Clin Exp Immunol. 1989;77:373-7.

19. Uma Devi KR, Ramalingam B, Brennan PJ, Narayanan PR, Raja A. Specific and early detection of $\operatorname{lgG}$, IgA and IgM antibodies to Mycobacterium tuberculosis 38-kDa antigen in pulmonary tuberculosis. Tuberculosis. 2001;81: 249-53.

20. Peirs $P$, Lefevre $P$, Boarbi $S$, Wang XM, Denis $O$, Braibant $M$, et al. Mycobacterium tuberculosis with disruption in genes encoding the phosphate binding proteins PstS1 and PstS2 is deficient in phosphate uptake and demonstrates reduced in vivo virulence. Infect Immun. 2005;73: 1898-902.

21. Lamarche MG, Wanner BL, Crépin S, Harel J. The phosphate regulon and bacterial virulence: a regulatory network connecting phosphate homeostasis and pathogenesis. FEMS Microbiol. 2008:32:461-73.

22. Pennini ME, Pai RK, Schultz DC, Boom WH, Harding CV. Mycobacterium tuberculosis 19-kDa lipoprotein inhibits IFN- $\gamma$-induced chromatin remodeling of MHC2TA by TLR2 and MAPK signaling. J Immunol. 2006;176: 4323-30.

23. Målen H, Berven FS, Søfteland T, Arntzen M $\varnothing$, D'Santos CS, De Souza GA, et al. Membrane and membrane-associated proteins in triton X-114 extracts of M. bovis/BCG identified using a combination of gel-based and gel-free fractionation strategies. Proteomics. 2008:8(9):1859-70.

24. Karbalaei Zadeh Babaki Z, Soleimanpour S, Rezaee SA. Antigen 85 complex as a powerful Mycobacterium tuberculosis immunogen: Biology, immunepathogenicity, applications in diagnosis, and vaccine design. Microb Pathog. 2017;112:20-9.

25. Gaur RL, Ren K, Blumenthal A, Bhamidi S, Gonzáles-Nilo FD, Jackson M, Zare $\mathrm{RN}$, et al. LprG-mediated surface expression of lipoarabinomannan is essential for virulence of Mycobacterium tuberculosis. PLoS Pathog. 2014; 10(9):e1004376. https://doi.org/10.1371/journal.ppat.1004376.

26. Gehring AJ, Dobos KM, Belisle JT, Harding CV, Boom WH. Mycobacterium tuberculosis LprG (Rv1411c): a novel TLR-2 ligand that inhibits human macrophage class II MHC antigen processing. J Immunol. 2004;173:2660-8.

27. Rezwan M, Laneelle MA, Sander P, Daffe M. Breaking down the wall. Fractionation of mycobacteria. J Microbiol Methods. 2007;68:32-9.

\section{Publisher's Note}

Springer Nature remains neutral with regard to jurisdictional claims in published maps and institutional affiliations.
Ready to submit your research? Choose BMC and benefit from:

- fast, convenient online submission

- thorough peer review by experienced researchers in your field

- rapid publication on acceptance

- support for research data, including large and complex data types

- gold Open Access which fosters wider collaboration and increased citations

- maximum visibility for your research: over $100 \mathrm{M}$ website views per year

At $\mathrm{BMC}$, research is always in progress.

Learn more biomedcentral.com/submissions 